\title{
Bilateral V-Y advancement gluteal fold flaps following vulvo-vaginal melanoma resection
}

\author{
Vlad Pieptu ${ }^{1}$, Alexandru Mihai ${ }^{1}$ Nicolae Ghetu ${ }^{*, 1,2}$, Nicolae Ioanid ${ }^{3}$, Dan Ferariu ${ }^{4}$, \\ Lucian Miron ${ }^{2,5}$, Vladimir Poroch ${ }^{2,6}$, Codrin Nicolae Dobreanu ${ }^{1}$
}

${ }^{1}$ Department of Plastic and Reconstructive Surgery, Regional Institute of Oncology laşi, Romania; 2“Grigore T. Popa” University of Medicine and Pharmacy, laşi, Romania; ${ }^{3}$ Department of Gynaecologic Oncological Surgery, Regional Institute of Oncology laşi, Romania; ${ }^{4}$ Department of Pathology, Regional Institute of Oncology laşi, Romania; ${ }^{5}$ Department of Oncology, Regional Institute of Oncology laşi, Romania; ${ }^{6}$ Department of Palliative care, Regional Institute of Oncology laşi, Romania.

\begin{abstract}
The soft tissue defects of the anterior perineum can be caused by tumour excision, trauma, burns, Fournier's gangrene and genital mutilation. Various reconstruction methods were described: vacuum-assisted closure, skin grafts and skin substitutes, pedicled or free flaps. In the case of defects following oncologic surgery, flaps are the reconstructive method of choice. We present the case of a 65 year-old woman diagnosed with stage III A vulvovaginal melanoma affecting also the distal urethra. Radical vulvectomy and en-bloc excision of the anterior third of the vagina and distal urethra, along with bilateral inguinal lymphadenectomy was performed. The resulting defect was covered with bilateral V-Y advancement gluteal fold flaps. A local vaginal recurrence appeared 18 months after surgery and was safely removed. The patient followed chemotherapy with Dacarbazine. The gluteal fold flap offered good defect coverage and survived completely. Follow-up period was two years without distant metastases. Functional result was excellent, urinary function being normal and patient satisfaction high. Due to the gluteal flap's advantages (appropriate thickness, good vascularity, minimal donor site morbidity, and same operative field allowing for shorter operating time), reconstruction of the anterior perineum after oncological resection with bilateral $\mathrm{V}-\mathrm{Y}$ advancement gluteal flaps is a viable solution.
\end{abstract}

Keywords: vulvar melanoma, perineum reconstruction, gluteal fold flap, melanoma

\section{Introduction}

The soft tissue defects of the anterior perineum can be caused by tumour excision, trauma, burns, Fournier's gangrene and genital mutilation. Various reconstruction

Received: August 2015; Accepted after review: September 2015; Published: September 2015.

${ }^{*}$ Corresponding author: Nicolae Gheţu, MD, PhD, Department of Plastic and Reconstructive Surgery, Regional Institute of Oncology laşi, Grigore T. Popa University of Medicine and Pharmacy, 2-4, Gen. Henri Mathias Berthelot St., 700483 Iaşi, Romania Email:dr.ghetu@gmail.com methods were described: vacuum-assisted closure (VAC) [1], skin grafts [2], skin substitutes [3], pedicled [4] or free flaps [5]. In the case of defects following oncologic surgery, flaps are the reconstructive method of choice because of defect size, the need to separate intraperitoneal contents from perineum and fill/obliterate dead space, and frequent subsequent radiotherapy [6].

Successful reconstruction of the female anterior perineum after oncologic resections is paramount for restoring genitourinary function and preserving body image and psychological well-being, thus improving quality of life. 


\section{Case report}

A 65 year-old woman was admitted to the Regional Oncological Institute lasi for postmenopausal bleeding. Upon inspection, an irregular-shaped hyperpigmented lentiginous lesion was identified, covering the anterior third of the vagina, urethral orifice, both labia and the clitoris (Figure 1). Microscopical analysis of a biopsy prelevated from the left labia minor showed ulcerative nodular malignant melanoma, with a Clark IV score and $5 \mathrm{~mm}$ Breslow. PAP smear showed no endocervical lesions.

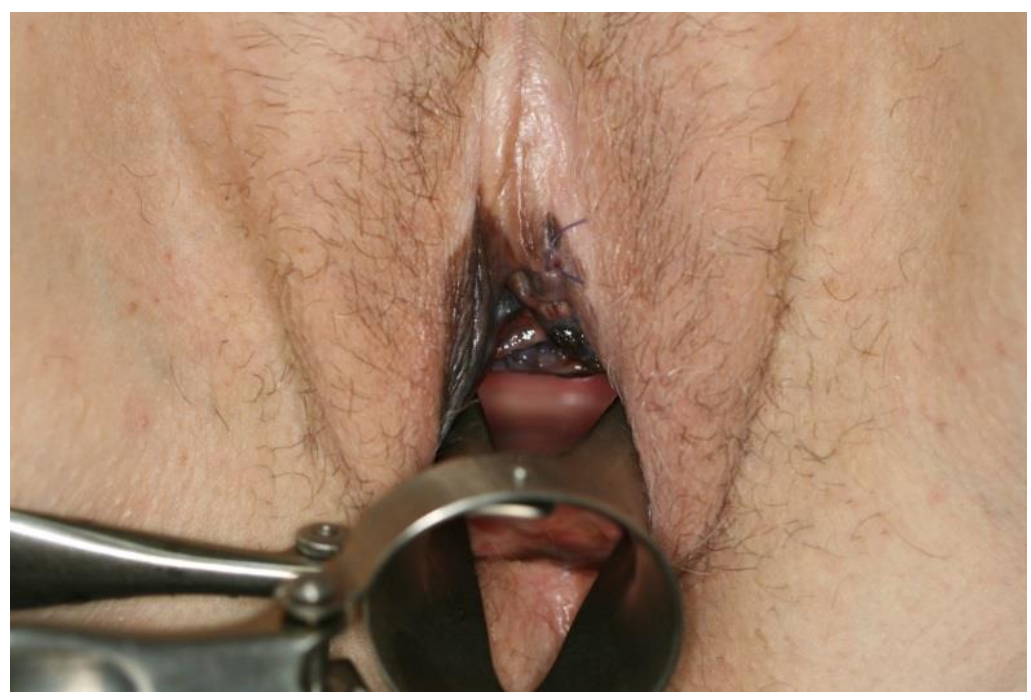

Fig. 1. Aspect upon admission. Extensive vulvo-vaginal melanoma affecting both labia, urethral orifice, clitoris and external vagina. Biopsy site on the upper left labia

The Oncologic Board decided for wide margin resection of the tumour, bilateral inguinal lymphadenectomy and simultaneous reconstruction; further treatment and follow-up plan was established upon histology results.

The posterior labial arteries and internal pudendal artery perforators (IPAP) were located using a hand-held bidirectional ultrasound Doppler (Hadeco ${ }$ Bidop ${ }^{\mathrm{TM}}$ ES$100 \mathrm{~V} 3$ with an $8 \mathrm{MHz}$ probe) and identified perforators were marked with a surgical pen (Figure 2).

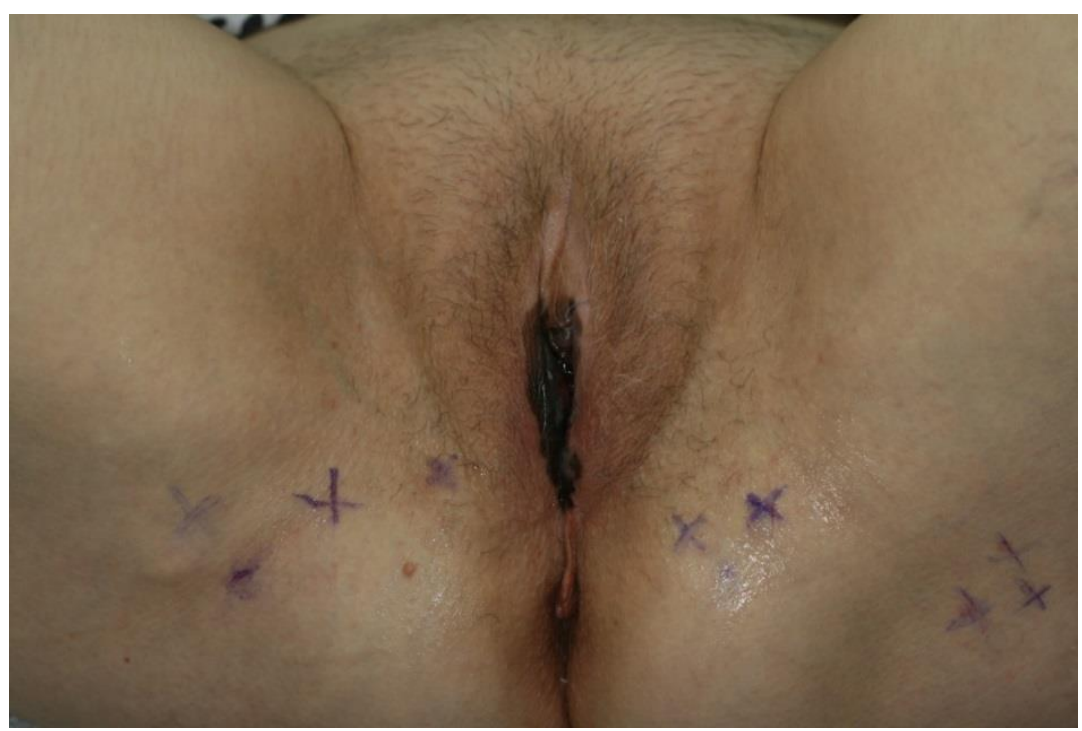

Fig. 2. Preoperative markings of the posterior labial arteries and internal pudendal artery perforators after Doppler perforator identification 
Bilateral superficial and deep inguinal lymph node resection (Figure 3) was performed, followed by radical vulvectomy with en-bloc excision of the anterior third of the vagina and distal urethra (Figure 4). The resulting defect measured 12/8 cm (Figure 5).
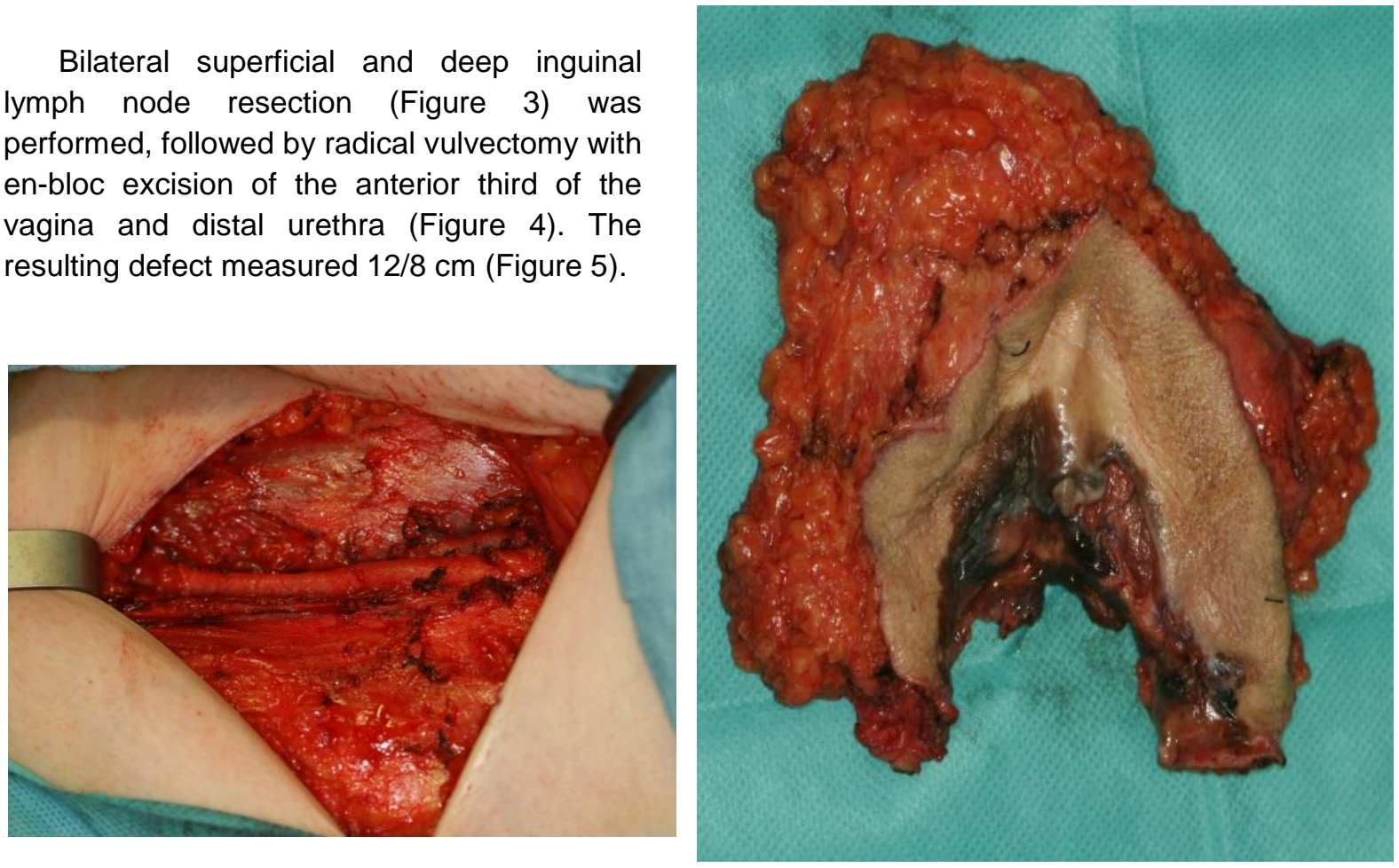

Fig. 3. Intraoperative aspect after superficial and deep inguinal lymph node resection: femoral artery and underneath it, femoral vein.

Fig. 4. Resection specimen: $11.5 \times 9.5 \times 3 \mathrm{~cm}$ containing vulva, lower third of vagina, both labia, clitoris, distal urethra and adjacent subcutaneous tissue. Note $5 \times 4.5 \times$ $0.5 \mathrm{~cm}$ dark brown ulcerated area, identified after microscopical analysis as mixed superficial spreading and nodular melanoma.

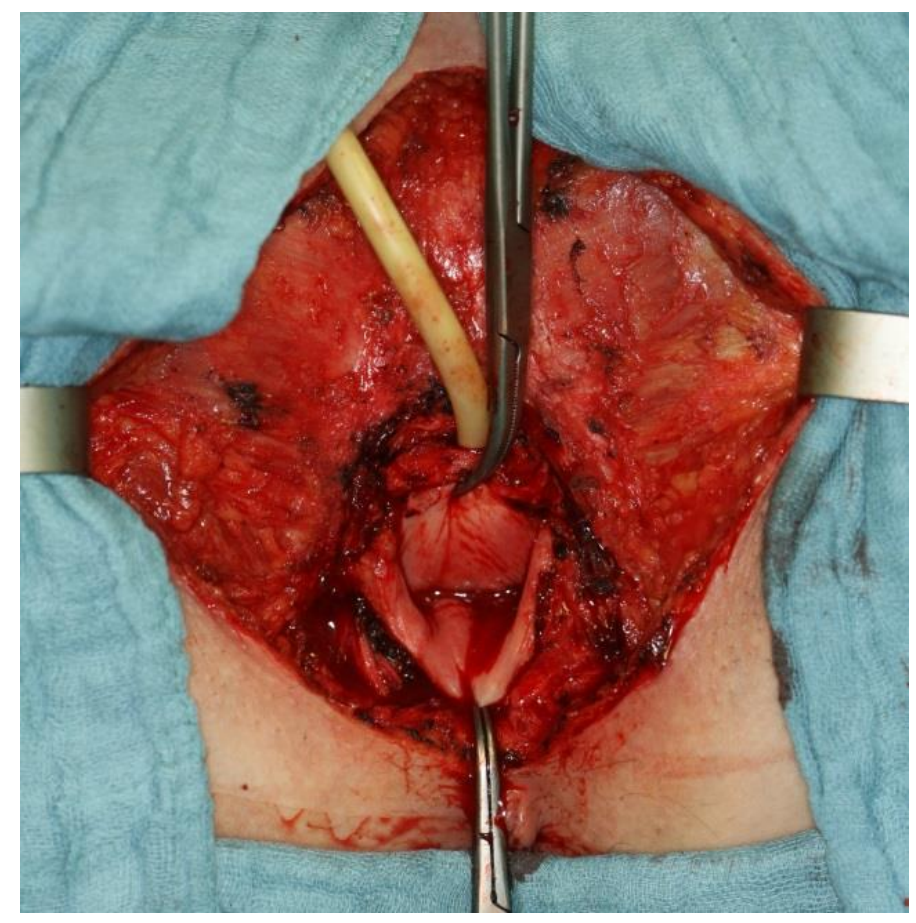

Fig. 5. Resulting defect after excision shows the vaginal stump, cannulated urinary tract and the $12 \times 8 \mathrm{~cm}$ area requiring coverage 
Bilateral V-Y advancement flaps were designed based on the internal pudendal artery perforators (Figure 6). The two $25 \times 15$ $\mathrm{cm}$ flaps were dissected in the plane of
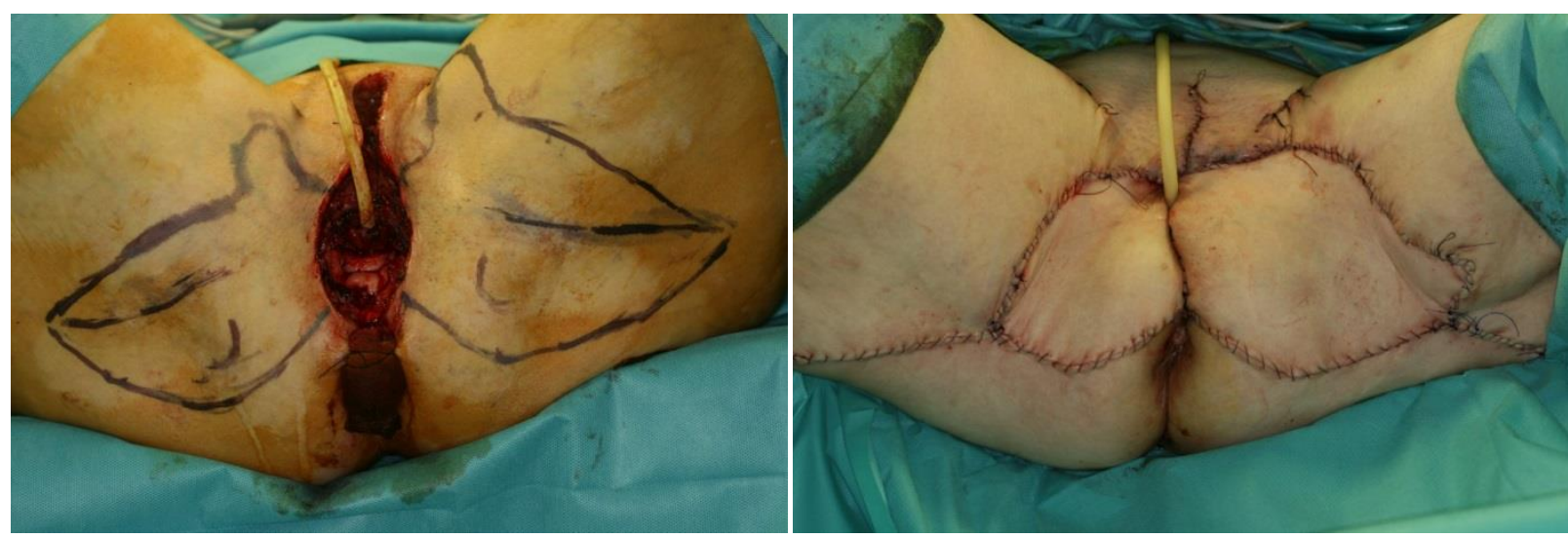

Fig. 6. Design of $\mathrm{V}-\mathrm{Y}$ advancement gluteal flaps

Postoperative care included daily dressings, Foley catheter, antidiarrheal drugs, alternate lateral decubitus for 3 weeks and refraining from sitting position, because direct pressure on the perineum risks compromising the flap vascularisation [7]. As the medial portions of the flaps have been sutured to the middle third of the vagina, the moist environment caused wound healing to be delayed by 5 days. The post-operative course was uneventful and the patient was discharged after one week. Gradual tolerance to sitting was introduced at the beginning of second week. Proprioceptive and protopathic sensitivity started to reappear at postoperative month 3 and further improved during the first postoperative year, reaching expected levels.

Microscopical analysis of the resection specimens showed positive margins at the superficial layers of the vaginal mucosa, other margins being at least $4.5 \mathrm{~mm}$ clear. One lymph node was positive for extension of the tumour. The remnant urethra was free of tumoral cells. According to AJCC, staging was T2N1M0 stage IIIA vulvo-vaginal melanoma. The Oncological Board re-evaluated the case and decided, according to patient's options, muscular fascia and advanced medially to cover the defect (Figure 7). Only minor skeletonization of the internal pudendal artery perforator was required, limiting total operation time to 4 hours.
Fig. 7. Immediate postoperative view of reconstructed area refraining from secondary surgical intervention and starting adjuvant chemotherapy with Dacarbazine.

The patient returned three weeks after the intervention because of a $2 \times 1 \mathrm{~cm}$ periurethral dehiscence, due to a urinary tract infection (UTI) caused by the Foley catheter. The dehiscence healed secondarily with daily dressings and antiseptic solution.

A local vaginal recurrence appeared at 18 months following initial surgery. Excision of the recurrence under local anaesthesia was performed, but the microscopical examination of the specimen revealed a positive margin. A second excision was performed and confirmed to be oncologically safe.

Two years after initial surgery there was no evidence of another local recurrence or metastases. The bilateral gluteal flaps were well integrated in the recipient area, no scar contractures were present and the skin was soft and pliable (Figure 8). The patient had no urinary complaints. She was satisfied with the reconstructive result, mainly because of the ability to return to her daily routine without further complications. 


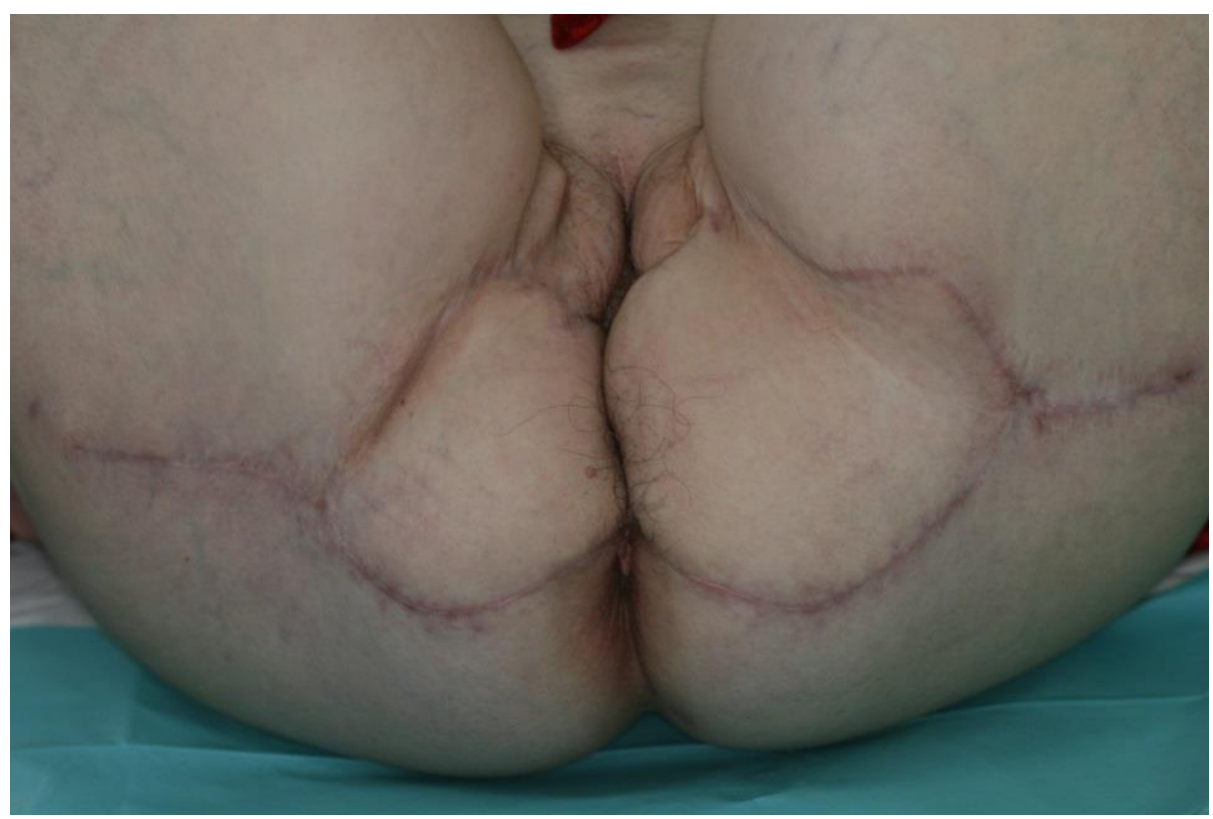

Fig. 8. Aspect at 2 years after reconstruction. Note lack of scar contracture, good colour and texture matching, along with overall aesthetic result

\section{Discussion}

Malignant melanoma of the vulva and vagina accounts for less than $2 \%$ of melanomas among women [8, 9]. It can be represented by an irregular pigmented lesion with rapid growth. Upon consultation, patients have symptoms similar to other vulvar malignancies: presence of a mass, bleeding or itching. Histological analysis of a full-thickness biopsy confirms the diagnosis, identifies the subtype and classifies its staging with the Clark and Breslow systems. There are three histological subtypes of vulvar melanoma: superficial spreading, nodular and acral lentiginous. It appears that nodular vulvar melanoma has a worse prognosis than the other two subtypes [10] but this has not been confirmed by other studies [11]. The median age at diagnosis is 66 years for vulvar melanoma and 70 years for vaginal melanoma [10]. Median survival rate is 61 months with $60 \%$ survival rate at 5 years and $50 \%$ survival rate at 10 years [10]. Patients with lateralized tumours have a better survival than those with clitoral or central tumours [10,11]. Prognosis is affected by age at discovery [12], histological subtype [13], Clark and Breslow staging, presence of ulceration [14], location of primary site and lymphatic space invasion [15].

Recommended treatment for vulvar and vaginal melanomas is surgical resection of the primary tumour, along with en-bloc bilateral femoral lymphadenectomy [16]. Although studies have not found correlation between extent of surgery (local excision, simple vulvectomy, radical vulvectomy, partial vaginectomy, radical vaginectomy, exenteration) and overall survival rate [17, 18], it is agreed that the resection specimen should have at least $1 \mathrm{~cm}$ clear margins [19].

Adjuvant radiotherapy, chemotherapy and immunotherapy have not yet shown to confer better survival rates. In salvage therapy, the most effective chemotherapeutic agent is Dacarbazine, with response rates varying from $15 \%$ to $25 \%$ [20].

Oncologic surgery of the vulva and vagina results in extensive soft-tissue defects. Due to the size of such defects and poor general status of the oncological patient, the use of pedicled flaps emerged as the preferred reconstruction method. For large volume defects, occurring after pelvic exenteration, bulky flaps such as vertical rectus abdominis muscle (VRAM), gracilis, omentum are 
preferred, to fill the dead space and separate the abdominal contents from the pelvic cavity $[6,21]$. For superficial and limited volume defects fasciocutaneous flaps like gluteal, lotus, IGAP are preferred [21, 22].

Introduced by Lee et al. in 2006 [23], the gluteal fold $V-Y$ advancement flap is vascularised by underlying fascial plexus originating from perforators of the internal pudendal artery combined with musculocutaneous perforators of underlying muscle. Innervation arises from the posterior cutaneous nerve of the thigh and the pudendal nerve. It has a triangular shape, the base of the flap is represented by the post-excisional wound margin and the apex is situated on the gluteal fold. Raising the flap does not require changing patient's position on the operating table after the resection, neither dissection of the perforators from the internal pudendal artery and the rami of pudendal nerve, thus lowering operating time and preserving sensation.

Due to the orientation of the long axis of the flap towards the gluteal crease, resulting scars are either covered by natural skin creases or placed on the uppermost inner thigh, thus being aesthetically acceptable. Considering that the donor site is adjacent to the recipient, the flap presents matched skin colour and texture. Furthermore, it is relatively thin even in overweight patients.

A small disadvantage is the fact that hairy skin from the remaining labia majora is introduced into the vaginal wall, but this does not produce an aesthetic or functional deficit. Patient compliance can be an issue, as the sitting position is to be avoided for a long

\section{References}

1. Schaffzin DM, Douglas JM, Stahl TJ, Smith LE. Vacuum assisted closure of complex perineal wounds. Dis Colon Rectum 2004; 47(10):17451748.

2. McAninch JW. Management of genital skin loss. Urol Clin North Am 1989; 16(2):387-397.

3. Payne CE, Williams AM, Hart NB. Lotus petal flaps for scrotal reconstruction combined with Integra resurfacing of the penis and anterior abdominal wall following necrotizing fasciitis. $J$ period. Lack of compliance can be prevented by careful patient selection combined with an explicit informed consent and counselling.

Complications are minor, wound dehiscence caused by patient non-compliance to postoperative movement restrictions or poor local hygiene being most frequent, followed by seromas or hematomas. These can be observed during daily dressing changes and leave little or no morbidities if treated early.

The main advantages of this flap are its vascular reliability, preservation of sensitivity, reduced thickness and texture matching. Adding to this the simple surgical technique, large available surface, reduced operating time and concealed scars, the $V-Y$ advancement gluteal flap is a noteworthy choice in the armamentarium of the reconstructive surgery of the perineum.

\section{Conclusion}

Bilateral V-Y advancement gluteal flaps are a viable solution for perineal reconstruction in patients who have suffered radical vulvectomy and partial vaginectomy. This reconstructive option allows for reliable tissue coverage with short operative times, minimal donor site morbidity and good long-term results, provided that oncologic status is under control.

\section{Conflict of interest}

Authors declare no conflicts of interest.

Plast Reconstr Aesthet Surg 2009; 62(3):393397.

4. Butler CE, Gundesliogu AO, Rodriguez-Bias MA. Outcomes of immediate vertical rectus abdominis myocutanous flap reconstruction for irradiated abdominoperineal defects. J Am Coll Surg 2008; (206):694-703.

5. Kieran I, Nugent N, Riordain MO, Kelly J. Reconstruction of the pelvis and perineum with a free latissimus dorsi myocutaneous flap: a 
case report. Ann $R$ Coll Surg Engl 2012; 94(8): e254-e256.

6. Salgado CJ,Chim H, Skowronski PP, Oeltjen J, Rodriguez M, Mardini S. Reconstruction of acquired defects of the vagina and perineum. Semin Plast Surg 2011; 25:155-162.

7. Pantelides NM, Davies RJ, Fearnhead NS, Malata CS. The gluteal fold flap: A versatile option for perineal reconstruction following anorectal cancer resection J Plast, Reconstr Aesthet Surg 2013; 66:812-e820.

8. Weinstock MA. Malignant melanoma of the vulva and vagina in the United States: Patterns of incidence and population-based estimates of survival. Am J Obstet Gynecol 1994; 171:12251230.

9. Ragnarsson-Olding $B$, Johansson $H$, Rutqvist LE, et al: Malignant melanoma of the vulva and vagina. Cancer 1993; 71:1893-1897.

10. Podratz KC, Gaffey TA, Symmonds RE, et al. Melanoma of the vulva: An update. Gynecol Oncol 1983; 16:153-168.

11. Scheistroen M, Trope $C$, Koern J, et al. Malignant melanoma of the vulva: evaluation of prognostic factors with emphasis on DNA poloidy in 75 patients. Cancer 1995; 75:72-80.

12. Rose PG, Piver MS, Tsukada $Y$, et al. Conservative therapy for melanoma of the vulva. Am J Obstet Gynecol 1988; 159:520525.

13. Bradgate MG, Rollason TP, McConkey CC, et al. Malignant melanoma of the vulva: $A$ clinicopathological study of 50 women. $\mathrm{Br} \mathrm{J}$ Obstet Gynecol 1990; 97:124-133.

14. Tasseron EWK, van der Esch EP, Hart AAM, et al. A clinicopathological study of 30 melanomas of the vulva. Gynecol Oncol 1992; 46:170-175.
15. Phillips GL, Bundy BN, Okagaki $T$, et al. Malignant melanoma of the vulva treated by radical hemivulvectomy: A prospective study of the Gynecologic Oncology Group. Cancer 1994; 73:2626-2632.

16. Trimble EL. Melanomas of the vulva and vagina. Oncology 1996; 10(7):1017-23; discussion 1024.

17. Davidson T, Kissin M, Westbury G: Vulvovaginal melanoma-should radical surgery be abandoned? Br J Obstet Gynecol 1987; 94:473-476.

18. Reid GC, Schmidt RW, Roberts JA, et al. Primary melanoma of the vagina: A clinicopathologic analysis. Obstet Gynecol 1989; 74:190-199.

19. Veronesi U, Cascinelli N. Narrow excision (1 $\mathrm{cm}$ margin): A safe procedure for thin cutaneous melanoma. Arch Surg 1991; 126:438-441.

20. Pritchard KI, Quirt IC, Cowan DH, et al. DTIC therapy in metastatic malignant melanoma: A simplified dose schedule. Cancer Treat Rep 1989; 64:1123.

21. Mughal M, Baker RJ, Muneer A, Mosahebi $A$. Reconstruction of perineal defects. Ann $R$ Coll Surg Engl 2013; 95:539-544.

22. Niranjan NS. Perforator flaps for perineal reconstruction. Semin Plast Surg 2006; 20(2):133-144.

23. Lee PK, Choi MS, Ahn ST, et al. Gluteal fold V$Y$ advancement flap for vulvar and vaginal reconstruction: A new flap. Plast Reconstr Surg $2006 ; 118(2): 401-406$. 